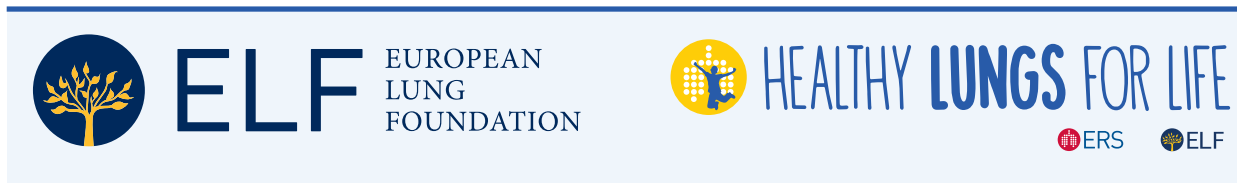

\title{
Your lungs and exercise
}

Regular physical activity and exercise improves quality of life, whether you are healthy or you have a lung condition. Many people associate keeping fit with maintaining a healthy heart, losing weight and reducing the risk of illnesses such as diabetes, but exercise also helps keep lungs healthy.

This factsheet explains how exercise affects the lungs, how breathing is influenced by activity and the benefits of exercise for people with and without a lung condition.

\section{What is exercise and how much should I do?}

Any type of physical activity counts as exercise. It could be planned sport such as running, swimming, tennis or bowls, an exercise training programme, or a hobby such as cycling or walking.

It could also include physical activities which are part of your daily life, such as gardening, cleaning or walking to the shops.

In order to stay healthy, you should do 30 minutes of moderate exercise five days per week. For a healthy person, moderate exercise might be walking at a pace of 4 to $6 \mathrm{~km}$ per hour. If you have a lung problem, you would need to walk fast enough to make you moderately breathless.

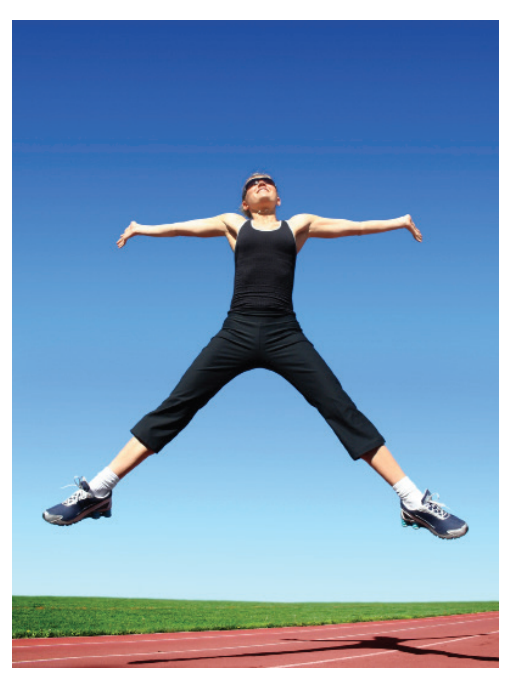

\section{Dhat happens to my lungs when I exercise?}

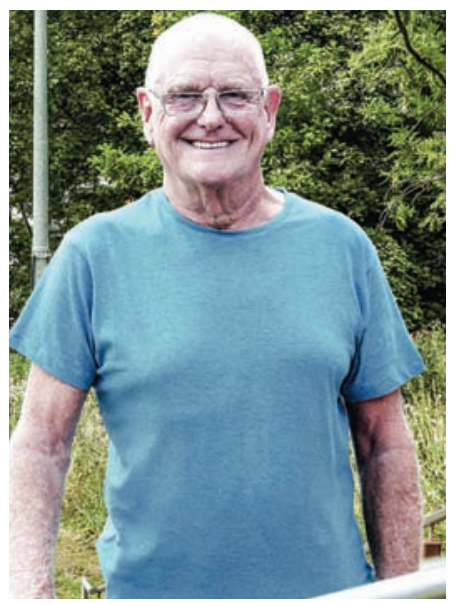

During exercise, two of the important organs of the body come into action: the heart and the lungs. The lungs bring oxygen into the body, to provide energy, and remove carbon dioxide, the waste product created when you produce energy. The heart pumps the oxygen to the muscles that are doing the exercise.

When you exercise and your muscles work harder, your body uses more oxygen and produces more carbon dioxide. To cope with this extra demand, your breathing has to increase from about 15 times a minute (12 litres of air) when you are resting, up to about 40-60 times a minute (100 litres of air) during exercise. Your circulation also speeds up to take the oxygen to the muscles so that they can keep moving.

When your lungs are healthy, you keep a large breathing reserve. You may feel 'out of breath' after exercise, but you will not be 'short of breath'. When you have reduced lung function, you may use a large part of your breathing reserve. This may make you feel 'out of breath', which can be an unpleasant feeling, but it is not generally dangerous. 
It is normal to get breathless during exercise. However, regular exercise can increase the strength and function of your muscles, making them more efficient. Your muscles will require less oxygen to move and they will produce less carbon dioxide. This will immediately reduce the amount of air you will need to breathe in and out for a given exercise. Training also improves your circulation and strengthens your heart.

Exercise will improve your overall physical and psychological well-being. It can decrease the risk of developing other conditions such as stroke, heart disease and depression. Regular exercise is also one of the most important interventions to prevent the onset of type-II diabetes.

\section{What can I do to help my lungs cope with exercise?}

The most important thing to do to keep your lungs healthy is to look after them. Smoking will affect your ability to undertake physical activity and reach your true potential. If you quit smoking, you are likely to be able to exercise for longer as early as two weeks after your last cigarette. The ELF factsheet on 'Smoking and the lungs' can provide more information on this topic.

\section{Dan exercise cause any problems with the lungs?}

People who follow high-intensity training regimes, or who regularly train in certain environments, may be at risk of developing exercise-induced asthma, or a condition known as bronchial hyperresponsiveness in which the airways become blocked after exercise. If take bronchodilators, you should normally take these 10 minutes before you exercise to prevent exercise-induced bronchoconstriction. Your doctor can give you advice on this.

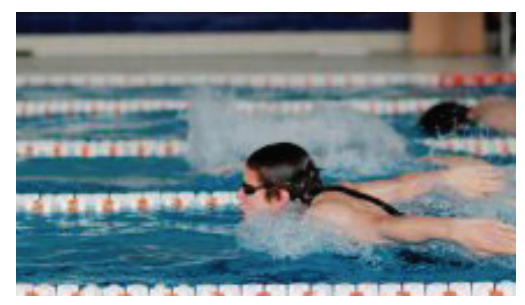

Scientists believe this is caused by harmful substances in the environment in which they are training, such as chlorine in a swimming pool, or cold dry air on a ski slope. Endurance athletes have the potential to inhale more harmful substances into their lungs, as they are exposed to these conditions for longer periods of time. Our factsheet, 'Asthma in elite athletes', provides more information on this.

\section{If I notice a problem, what should I do?}

It is important that you are aware of the symptoms associated with lung problems, such as cough, shortness of breath or fatigue, and that you see your doctor about them as soon as possible.

When you visit the doctor, you may be asked to take a spirometry test. This tests your breathing and can help in the diagnosis of lung problems. You will be asked to breathe into a device that measures the amount of air in your lungs and how fast you can breathe out. Our spirometry factsheet provides more information on the test. You may also be asked to take an exercise test to measure your limitations.

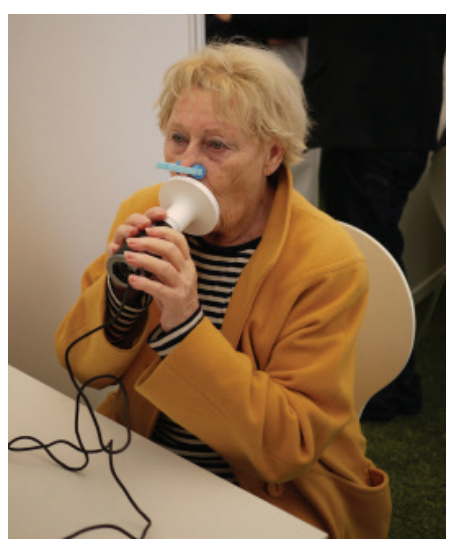




\section{Exercising with a long-term lung condition}

People with long-term lung conditions can help improve their symptoms through regular exercise.

If you have a long-term lung condition, the thought of becoming quickly out of breath can be daunting and you may not feel motivated to exercise. It can be tempting to avoid exercise because you think it will make you breathless, but if you do less activity you become less fit and daily activities will become even harder.

It is best to ask the guidance of a doctor or physiotherapist before you begin exercising, to ensure that your exercise plans are in line with your capacity and are safe. All exercise programmes must be built up over time to allow the body to adapt. It's important that you exercise at your own pace. If you reach a stage where you are too breathless to talk, then slow down the pace or if necessary take a short pause. The more you do, the more you will be able to do!

Intermittent exercises can also help you to deal with the shortness of breath. In this case you alternate brief exercise, lasting 1-2 minutes, with moments of rest (or slower exercise). This is called 'interval training'.

A If your breathlessness suddenly becomes worse or does not decrease rapidly after exercise, you should see a doctor.

\section{COPD}

If you have COPD, you will have damaged airways. This means that when you breathe out your airways become narrowed before you have got rid of all the air in your lungs. Many people with COPD find that pursing their lips enables them to breathe out more slowly and effectively. You may also find it easier to walk with braced arms (for example leaning on a shopping stroller, or even by grasping the belt of your trousers). Patients with severe lung disease can benefit from using a wheeled walking aid.

If you suffer from severe COPD, you may have problems bringing enough oxygen into the body. If this is the case, you may need to take supplementary oxygen during your activity. Your doctor will be able to assess this before you begin a training programme.

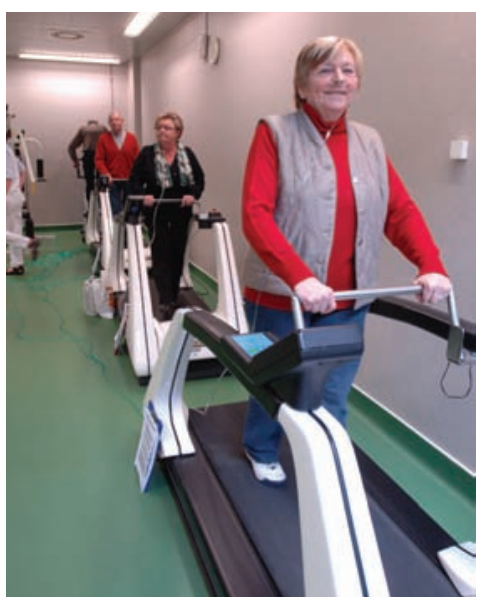

Read factsheet on 'Living an active life with COPD' for more information on how to stay active with COPD.

\section{Lung fibrosis}

If you suffer from lung fibrosis, interstitial lung diseases or problems with the chest wall, you will have difficulties expanding your lungs. You may need to breathe in more deeply and slowly, to allow the lungs to expand as much as possible. 


\section{Asthma}

Having asthma should not restrict your ability to exercise or be physically active. If you feel uncomfortable during or after exercise, you should ask your doctor to investigate whether the management of your condition could be improved. In fact, many athletes have asthma and are able to compete at the highest level when their condition is well-controlled.

Your doctor may prescribe medicine to control your symptoms. Inhaled steroids, a common type of drug used by people with asthma, are the most important controller medication you can take. $\beta 2$ agonists are the best medication to give quick short term relief from symptoms.

If you are an athlete hoping to take part in competitions, you should check whether your medication is listed as a performance-enhancing drug. Most asthma drugs (including inhaled steroids) have no restrictions, but it important to check each drug you are planning to take. By receiving the best treatment, as early as possible, you will have the best chance of competing on equal terms with nonasthmatic competitors.

Case study: Kjeld Hansen, Denmark, has asthma and completed the New York marathon.

"It is hard to motivate yourself to train when your 100\% is no match for a nonasthmatic person. My asthma sometimes felt as if I was breathing through a whistle. If you try to run and whistle at the same time, you'll feel what I felt."

"During training for the marathon, I felt my confidence grow. I followed my treatment regime carefully and exercised at least 3 times a week. By following my treatment plan, I was able to get rid of my asthma symptoms. The marathon training also felt amazing - I was able to push myself to new levels and see improvements day-by-day."

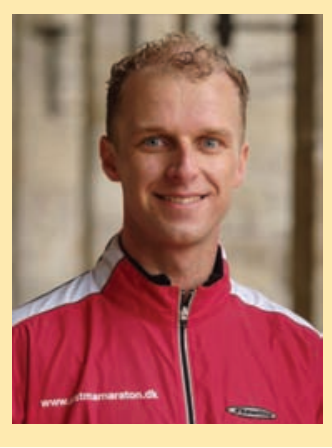

\section{Deneral tips}

- At the start of your work-out, prepare yourself with gentle activities involving the muscles you will be using duing your exercise (warm up)

- Improve your flexibility with stretching exercises

- Gradually improve your ability to exercise for longer periods (build up stamina)

- Increase activity at your own pace, and do not be afraid to get modestly out of breath (i.e. 4-5 on a scale of $0-10$ )

- Improve your muscle strength (i.e. by lifting weights)

- At the end of your work-out, slow down your activities and stretch the muscles you have used and allow your breathing to return to normal (cool down)

Remember: Exercise can bring many benefits and be enjoyable, even with a long-term health problem. Even if a task seems difficult at first, if you tackle one thing at a time at your own pace, you will quickly notice an improvement in your symptoms. together the leading European medical experts to provide patient information and raise public awareness about respiratory disease. 